\title{
On rational Morley triangles
}

\author{
by \\ ANDrew Bremner (Tempe, AZ), \\ Joseph R. Goggins (Girvan), \\ Michael J. T. GuY (Cambridge) \\ and Richard K. GuY (Calgary, Alta)
}

1. One of the great theorems of elementary plane geometry was essentially only discovered in the twentieth century; namely, the theorem of Morley that states that the trisectors of the angles of a triangle meet at the vertices of an equilateral triangle (the "Morley triangle"). Here, it is necessary to identify precisely the correct pairing of angle trisectors. It is the proximal trisectors that are involved. For example, in Figure 1, the line $A C 0$ making an angle $A / 3$ with $A C$, and the line $C A 0$ making an angle $C / 3$ with $C A$ intersect at the point $0 * 0$; and similarly for $* 00,00 *$, with $* 000 * 000 *$ an equilateral triangle (the "canonical" Morley triangle). Of course, the line making an angle $(A+2 \pi) / 3$ with $A C$ is an equally valid trisector of angle $A$, as is the line making an angle $(A+4 \pi) / 3$ with $A C$. So there are three possible trisectors at each angle, yielding 27 intersection points. Surprisingly, these lie six on each of three sets of three parallel lines, so that there are an apparent 27 Morley triangles. However, 9 of them, which have been called the Guy Faux triangles, are generated by trisectors of only two of the three angles of the original triangle, leaving 18 genuine, pairwise homothetic, Morley triangles. The underlying geometry has recently been well described by John Conway, using his concept of extraversion, transforming a triangle $A B C$ into itself, leading to triangles with angles

$$
\begin{gathered}
A-i \pi, B-j \pi, C-k \pi, \quad \text { where } i+j+k=0, \text { or } \\
i \pi-A, j \pi-B, k \pi-C, \quad \text { where } i+j+k=2 .
\end{gathered}
$$

As we are concerned with trisection we work modulo 3 , and the number of distinct triples $i j k$ which satisfy these relations is $2 \cdot 3 \cdot 3=18$.

2000 Mathematics Subject Classification: Primary 51M04, 11D41.

R. K. Guy partially supported by Natural Sciences \& Engineering Research Council Grant A-4011. 
The general situation is illustrated in Figure 4. The 18 trisecting lines are labelled $X Y i$, with $X \neq Y \in\{A, B, C\}, i \in\{0,1,2\}$, being the trisector of angle $X+2 \pi i$ adjacent to the edge $X Y$ of the triangle. Trisectors $A B i$, $B A j$ intersect at $i j * ; B C j, C B k$ intersect at $* j k$, and $C A k, A C i$ intersect at $i * k$. The vertices of triangle $i j k$ are $* j k, i * k, i j *$, and each point is the vertex of just two triangles, whose labels are found by replacing the $*$ by those two values from $\{0,1,2\}$ which make $i+j+k \equiv 0$ or $2 \bmod 3$. The nine Morley lines are labelled $I J K$, with $I+J+K \equiv 1 \bmod 3$ and a point lies on a line just if $i \neq I, j \neq J, k \neq K$. E.g., the line 121 contains the six points $* 12,0 * 2,00 *, * 00,2 * 0,21 *$. The Guy Faux triangles are characterized by having the $*$ in the same respective position in the vertices: for example, the triangle with vertices $1 * 2,0 * 0,2 * 1$ (note that each of these points is a vertex of two genuine Morley triangles, for instance, $1 * 2$ being a vertex of the Morley triangles 102 and 122).

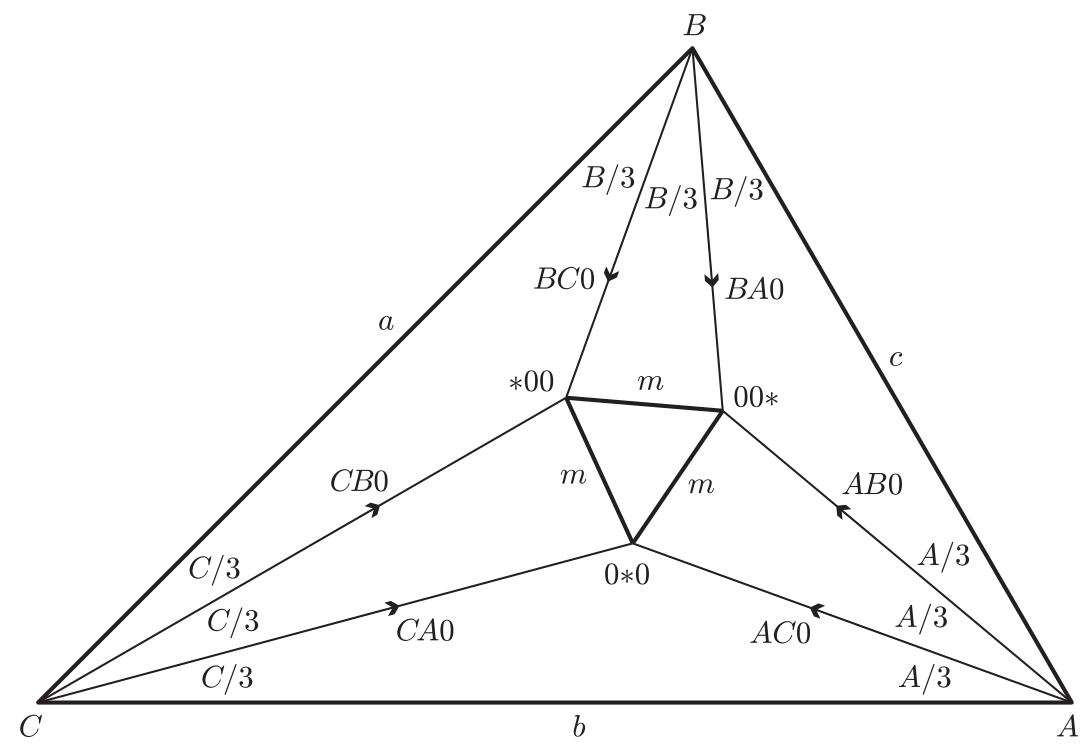

Fig. 1. The "canonical" Morley triangle, 000

The interest here lies in the arithmetic of the Morley triangle, and in particular, the determination of those triangles of rational edges ("rational triangles") which possess a Morley triangle of rational edge. For example, the isosceles triangle $(286,343,343)$ has the canonical Morley triangle of edge $294 / 5$; and the triangle $(748,2197,2401)$ has canonical Morley triangle of edge 1001/5. We show that such triangles are either equilateral (in which case six of the 18 Morley triangles are rational, and in fact congruent to the original triangle), or belong to a one-parameter family of Pythagorean triangles (in which case just two of the 18 Morley triangles are rational and 
equal in size), or else belong to a two-parameter family of triangles, with all 18 Morley triangles rational.

2. In Figure 1, let $m$ denote the edge length of the Morley triangle of the triangle $A B C$ with edges $a, b, c$. Denote the radius of the circumcircle of $A B C$ by $R$, and the area of $A B C$ by $\triangle$. The following are well known formulas (see, for example, [1, pp. 2, 3, 58, 50]):

$$
\begin{gathered}
a=2 R \sin A, \quad b=2 R \sin B, \quad c=2 R \sin C, \quad R=a b c /(4 \Delta), \\
16 \Delta^{2}=(a+b+c)(b+c-a)(c+a-b)(a+b-c), \\
m=8 R \sin A / 3 \sin B / 3 \sin C / 3 .
\end{gathered}
$$

Our convention is that when referring to an angle such as $A / 3$, it may refer to any one of the three trisectors of $A$ (though in any particular set of equations, the choice must be made consistently). The condition that the trisectors $A / 3, B / 3, C / 3$ do indeed give rise to a Morley triangle is

$$
\frac{A}{3}+\frac{B}{3}+\frac{C}{3} \equiv \pm \frac{\pi}{3} \bmod 2 \pi \text {. }
$$

THEOREM. If a rational edged triangle has a rational Morley triangle, then either the original triangle is equilateral (and 6 of the 18 Morley triangles are rational), or it is Pythagorean belonging to a one-parameter family (and 2 of the 18 Morley triangles are rational), or it belongs to a twoparameter family of triangles (and all 18 Morley triangles are rational).

Pro of. For a set of trisectors $A / 3, B / 3, C / 3$ satisfying (2), define

$$
u=2 R \sin A / 3, \quad v=2 R \sin B / 3, \quad w=2 R \sin C / 3 .
$$

Since $R^{2}$ is rational from (1), $u, v, w$ are algebraic numbers of degree at most 3 , satisfying the equations:

(4) $\quad u^{3}-3 R^{2} u+a R^{2}=0, \quad v^{3}-3 R^{2} v+b R^{2}=0, \quad w^{3}-3 R^{2} w+c R^{2}=0$.

From (1), the associated Morley triangle edge length is given by

$$
m=8 R \sin \frac{A}{3} \sin \frac{B}{3} \sin \frac{C}{3}=\frac{u v w}{R^{2}}
$$

and since $m \in \mathbb{Q}$ by assumption, we have necessarily

$$
u v w \in \mathbb{Q} \text {. }
$$

We treat first the case where the triangle possesses a right angle, say at $C$. Then $R= \pm c / 2$, and $m= \pm 2 c \sin A / 3 \sin B / 3$, necessarily forcing $\sin A / 3 \sin B / 3 \in \mathbb{Q}$. Suppose $u=2 R \sin A / 3$ is a cubic irrational, so that necessarily $v$ is a cubic irrational. Then $v=r / u, r \in \mathbb{Q}$, and (4) implies

$$
\frac{r^{3}}{u^{3}}-3 R^{2} \frac{r}{u}+b R^{2}=0, \quad \text { that is, } u^{3}-3 \frac{r}{b} u^{2}+\frac{r^{3}}{b R^{2}}=0 .
$$


But

$u^{3}-3 R^{2} u+a R^{2}=0, \quad$ implying that $\frac{3 r}{b} u^{2}-3 R^{2} u+\left(a R^{2}-\frac{r^{3}}{b R^{2}}\right)=0$, and $u$ is at most quadratic, a contradiction. Thus neither $u$ nor $v$ can be a cubic irrational. So both are at most quadratic, and the equations for $u, v$ at (4) each have at least one rational root, say $u_{0}, v_{0}$. Fix angles $A, B$ so that $2 R \sin A / 3=u_{0}$ and $2 R \sin B / 3=v_{0}$. Then $\sin A / 3, \sin B / 3 \in \mathbb{Q}$. However, the triplication formula $\cos A=(\cos A / 3)\left(1-4 \sin ^{2} A / 3\right)$ implies that $\cos A / 3=(\cos A) /\left(1-u_{0}^{2} / R^{2}\right)$, and hence $\cos A \neq 0$ implies that $\cos A / 3 \in \mathbb{Q}$. Similarly $\cos B / 3 \in \mathbb{Q}$. Putting $t=\tan A / 6 \in \mathbb{Q}$, it follows that $\sin A / 3=2 t /\left(1+t^{2}\right), \cos A / 3=\left(1-t^{2}\right) /\left(1+t^{2}\right)$. In turn,

$$
\cos A=\frac{\left(1-t^{2}\right)\left(1-14 t^{2}+t^{4}\right)}{\left(1+t^{2}\right)^{3}}, \quad \sin A=\frac{2 t\left(3-t^{2}\right)\left(1-3 t^{2}\right)}{\left(1+t^{2}\right)^{3}} ;
$$

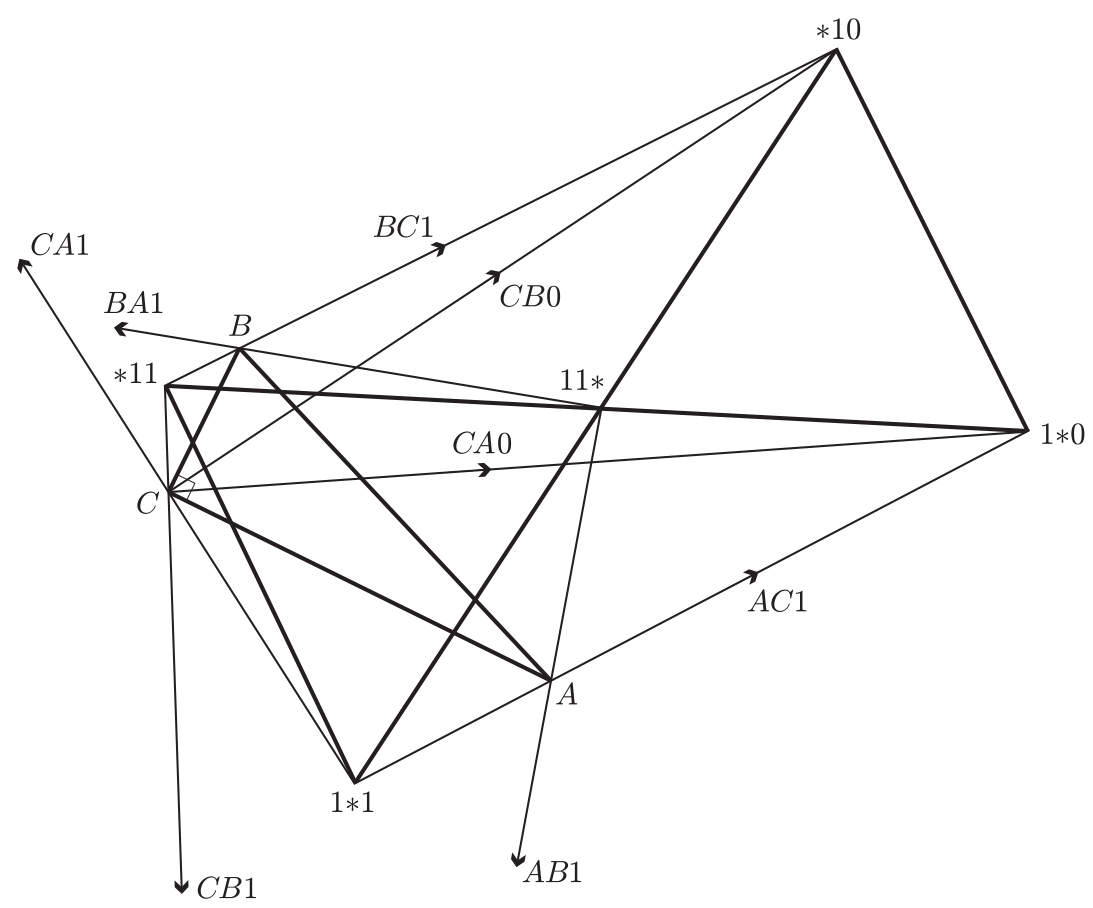

Fig. 2. The triangle $(44,117,125)$, right-angled at $C$, has two rational Morley triangles, 110 and 111, each with edge length 120

and since $A+B+C \equiv \pi \bmod 2 \pi$, we have $\cos B= \pm \sin A$, etc. The triangle up to scaling is thus parametrized by

$$
\{a, b, c\}=\left\{2 t\left(3-t^{2}\right)\left(1-3 t^{2}\right),\left(1-t^{2}\right)\left(1-14 t^{2}+t^{4}\right),\left(1+t^{2}\right)^{3}\right\}
$$


and it is now straightforward to compute that the edges of the 18 Morley triangles are the following:

$$
\begin{aligned}
& -4 t\left(1-t^{4}\right)(\text { twice }), \quad 2 t\left(1-t^{4}\right) \pm \frac{1}{2}\left(1+t^{2}\right)\left(1-6 t^{2}+t^{4}\right) \sqrt{3} \text { (each twice), } \\
& 2 t\left(1+t^{2}\right)\left(1-t^{2} \pm 2 t \sqrt{3}\right), \quad-4 t\left(1+t^{2}\right)\left(1-t^{2} \pm 2 t \sqrt{3}\right) \\
& \left(1-t^{4}\right)\left(2 t \pm\left(1-t^{2}\right) \sqrt{3}\right), \quad-2\left(1-t^{4}\right)\left(2 t \pm\left(1-t^{2}\right) \sqrt{3}\right) \\
& -4 t\left(1-t^{4}\right) \pm \frac{1}{2}\left(1+t^{2}\right)^{3} \sqrt{3}, \quad 8 t\left(1-t^{4}\right) \pm\left(1+t^{2}\right)^{3} \sqrt{3}
\end{aligned}
$$

Figure 2 illustrates the case $t=2$.

We treat second the case where the triangle does not have a right angle, and invoke the following lemma:

Lemma. Suppose that none of $A, B, C$ equals $\pm \pi / 2$. If each of the equations (4) has a root in the same algebraic number field $K$, then all the roots of each equation (4) lie in $K$.

Proof. Each of the equations (4) has a root in $K$, say $u_{0}, v_{0}, w_{0}$ respectively. Fix angles $A, B, C$ so that $2 R \sin A / 3=u_{0}, 2 R \sin B / 3=v_{0}$, $2 R \sin C / 3=w_{0}$. Using the triplication formula as in the Pythagorean case shows that $\cos A / 3$ lies in $K$; similarly for $\cos B / 3, \cos C / 3$. From (2) follows, on taking sine,

$$
\begin{aligned}
\sin \frac{A}{3}\left(\cos \frac{B}{3} \cos \frac{C}{3}-\right. & \left.\sin \frac{B}{3} \sin \frac{C}{3}\right) \\
& +\cos \frac{A}{3}\left(\sin \frac{B}{3} \cos \frac{C}{3}+\cos \frac{B}{3} \sin \frac{C}{3}\right)= \pm \frac{\sqrt{3}}{2}
\end{aligned}
$$

and multiplying by $R$,

$$
\frac{u_{0}}{2}\left(\cos \frac{B}{3} \cos \frac{C}{3}-\frac{v_{0} w_{0}}{4 R^{2}}\right)+\cos \frac{A}{3}\left(\frac{v_{0}}{2} \cos \frac{C}{3}+\frac{w_{0}}{2} \cos \frac{B}{3}\right)= \pm \frac{R \sqrt{3}}{2} .
$$

The left hand side here is now in $K$, and consequently $R \sqrt{3} \in K$. But

$$
\begin{aligned}
2 R \sin \left(\frac{A \pm 2 \pi}{3}\right) & =2 R\left(-\frac{1}{2} \sin \frac{A}{3} \pm \frac{\sqrt{3}}{2} \cos \frac{A}{3}\right) \\
& =-u_{0} / 2 \pm R \sqrt{3} \cos A / 3
\end{aligned}
$$

and lies in $K$; so the conjugates of $u_{0}$ all lie in $K$. Similarly, each equation at (4) has three roots in $K$.

As in the first case, we suppose again that at least one of $u, v, w$, say $u$, is a cubic irrational. From (6), at least one of $v, w$, say $v$, is a cubic irrational. Just as above, the case of $w$ rational is impossible, and so $w$ is either quadratic or cubic irrational. Suppose $w$ is quadratic irrational. Since $u v w \in \mathbb{Q}$, we have $\mathbb{Q}(w) \subseteq \mathbb{Q}(u, v)$, forcing the latter field to have 
degree 6 over $\mathbb{Q}$. This in turn implies that $\mathbb{Q}(u, v)$ has degree 2 over $\mathbb{Q}(u)$. Accordingly the cubic for $v$ at $(4)$ has a root in $\mathbb{Q}(u)$. Since the cubic for $w$ at (4) has one rational root, each of the equations at (4) has a root in $\mathbb{Q}(u)$. By the Lemma in the case $K=\mathbb{Q}(u)$, each of the equations at (4) splits over $\mathbb{Q}(u)$, so $\mathbb{Q}(u)=\mathbb{Q}(v)$ contradicting our deduction that $\mathbb{Q}(u, v)$ is quadratic over $\mathbb{Q}(u)$. Consequently, $w$ is a cubic irrational. The three conjugates of $u$ correspond to the three choices of trisector of angle $A$, so correspond to replacing $A / 3$ by $A / 3,(A \pm 2 \pi) / 3$; similarly for $v, w$. Thus the choice of trisectors $(A+2 \pi) / 3,(B+2 \pi) / 3,(C+2 \pi) / 3$ (satisfying (2)) leads to a Morley triangle of edge $m^{\prime}=u^{\prime} v^{\prime} w^{\prime} / R^{2}$ with $u^{\prime}, v^{\prime}, w^{\prime}$ conjugates of $u, v, w$. But $u v w \in \mathbb{Q}$, and so is fixed under conjugation, whence $m=m^{\prime}$ and $u v w=u^{\prime} v^{\prime} w^{\prime}$.

In this way

$$
\begin{aligned}
& \sin A / 3 \sin B / 3 \sin C / 3 \\
& \quad=\sin (A \pm 2 \pi) / 3 \sin (B \pm 2 \pi) / 3 \sin (C \pm 2 \pi) / 3
\end{aligned}
$$

(with signs respectively). If we use the identity

$$
\begin{aligned}
& 4 \sin \alpha / 3 \sin \beta / 3 \sin \gamma / 3 \\
& \quad=\sin (\delta-2 \alpha / 3)+\sin (\delta-2 \beta / 3)+\sin (\delta-2 \gamma / 3)-\sin \delta
\end{aligned}
$$

where $\delta=\alpha / 3+\beta / 3+\gamma / 3$, then (7) becomes

$$
\begin{aligned}
& \sin (D-2 A / 3)+\sin (D-2 B / 3)+\sin (D-2 C / 3) \\
& =\sin (D-2 A / 3 \pm 2 \pi / 3)+\sin (D-2 B / 3 \pm 2 \pi / 3)+\sin (D-2 C / 3 \pm 2 \pi / 3)
\end{aligned}
$$

(signs respectively), with $D=A / 3+B / 3+C / 3$; equivalently, by the "difference of sine" identity,

(9) $\cos (D-2 A / 3 \pm \pi / 3)+\cos (D-2 B / 3 \pm \pi / 3)+\cos (D-2 C / 3 \pm \pi / 3)=0$

(signs respectively). Adding and subtracting the two equations at (9) gives

$$
\begin{gathered}
\cos (D-2 A / 3)+\cos (D-2 B / 3)+\cos (D-2 C / 3)=0, \\
\sin (D-2 A / 3)+\sin (D-2 B / 3)+\sin (D-2 C / 3)=0 .
\end{gathered}
$$

Then

$$
\begin{aligned}
1 & =\cos ^{2}(D-2 C / 3)+\sin ^{2}(D-2 C / 3) \\
& =2+2 \cos (D-2 A / 3) \cos (D-2 B / 3)+2 \sin (D-2 A / 3) \sin (D-2 B / 3) \\
& =2+2 \cos (2 A / 3-2 B / 3) .
\end{aligned}
$$

Hence $\cos (2 A / 3-2 B / 3)=-1 / 2$, forcing $2 A / 3-2 B / 3 \equiv \pm 2 \pi / 3 \bmod 2 \pi$. So $A \equiv B \bmod \pi$, and by symmetry, $A \equiv B \equiv C \bmod \pi$; thus the triangle is equilateral. The edges of the 18 Morley triangles may be computed as having lengths -1 (with multiplicity 6 ), $\theta$ where $\theta^{3}-3 \theta^{2}+1=0$ (each root 


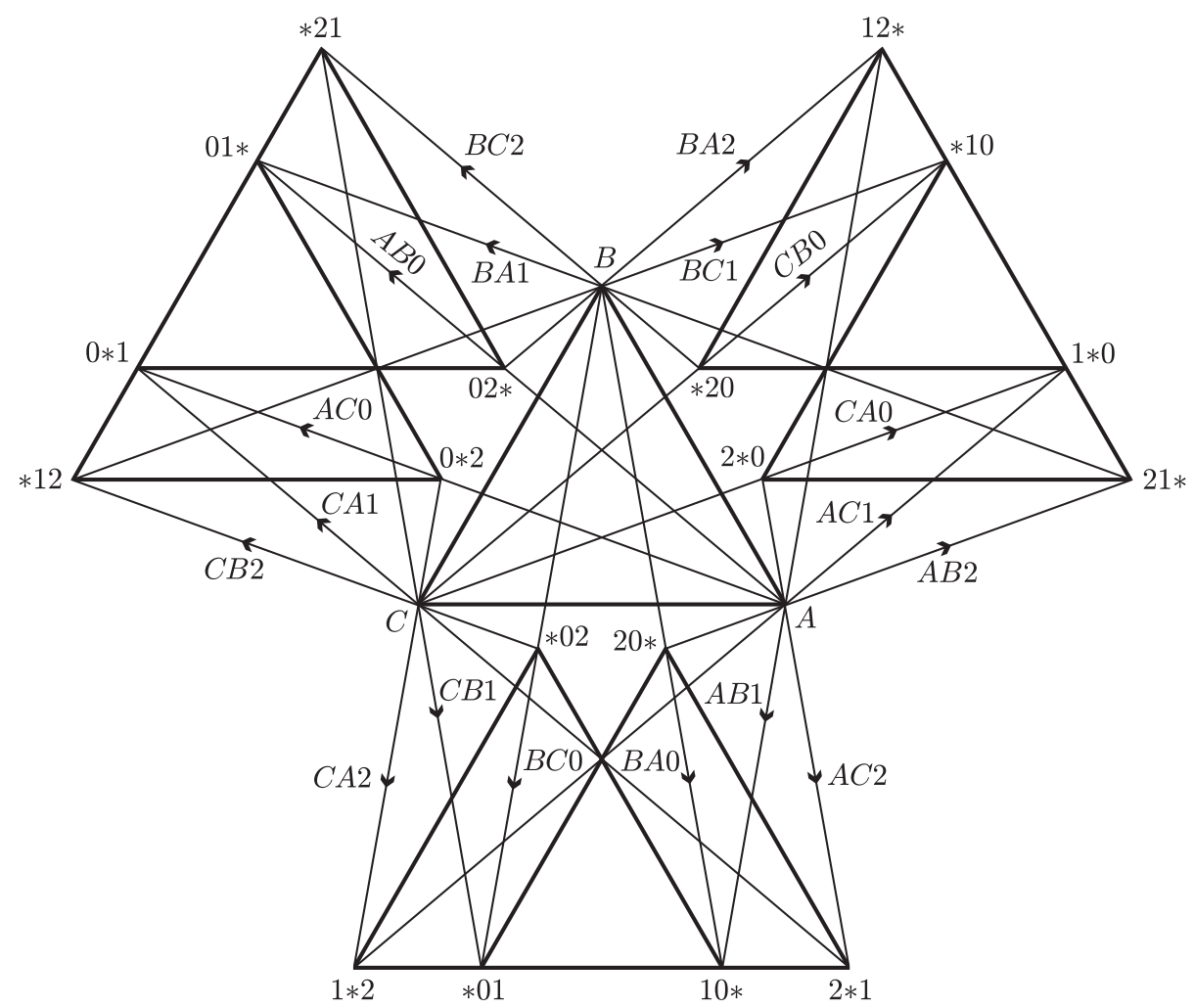

Fig. 3. An equilateral triangle has six of its Morley triangles, 021, 120, 012, 210, 102, 201, each congruent to the original triangle

with multiplicity 3 ), and $\phi$ where $\phi^{3}+3 \phi^{2}-6 \phi+1=0$ (each root with multiplicity 1).

Suppose finally that $u, v, w$ are at most quadratic irrationals. Then each of the equations (4) has a rational root, and by the Lemma in the case $K=\mathbb{Q}$, each equation at (4) has 3 rational roots. Thus every trisector gives rise to rational $u, v, w$ and all 18 Morley triangles are rational.

It is straightforward in this latter case to parametrize all rational triangles which have a rational Morley triangle. Note that such a triangle has the property that $\sin A / 3, \sin B / 3, \sin C / 3$ (and $R$ ) are each rational multiples of $\sqrt{3}$; and $\cos A / 3, \cos B / 3, \cos C / 3$ are rational. Now

$$
\tan ^{2} \frac{A}{6}=\frac{1-\cos A / 3}{1+\cos A / 3} \in \mathbb{Q}, \quad \text { and } \quad \sin \frac{A}{3}=\frac{2 \tan A / 6}{1+\tan ^{2} A / 6}
$$

implies that $\tan A / 6$ is a rational multiple of $\sqrt{3}$; similarly for $\tan B / 6$, $\tan C / 6$. 
Put $\tan A / 6=\alpha / \sqrt{3}, \tan B / 6=\beta / \sqrt{3}, \tan C / 6=\gamma / \sqrt{3}$ (accordingly, $\alpha, \beta, \gamma \in \mathbb{Q})$, and substitute into the equation resulting from (2):

$$
\tan (A / 6+B / 6+C / 6)= \pm 1 / \sqrt{3} .
$$

There results

$$
3(\alpha+\beta+\gamma)-\alpha \beta \gamma=\varepsilon(\beta \gamma+\gamma \alpha+\alpha \beta-3), \quad \varepsilon= \pm 1,
$$

and if we put

$$
(\alpha, \beta, \gamma)=\left(r, s, \frac{3(\varepsilon t-1)}{t+3 \varepsilon}\right)
$$

corresponding to $\tan (C+\varepsilon \pi) / 6=t / \sqrt{3}$, then (10) becomes

$$
3(r+s+t)=r s t .
$$

(Note that the transformation (11) fails to be invertible for $t$ precisely when $\gamma=3 \varepsilon$, that is, $\tan C / 6= \pm \sqrt{3}$. But now $C / 6 \equiv \pm \pi / 3 \bmod \pi$, so that $C \equiv 0 \bmod 2 \pi$, and the triangle is degenerate.)

Thus a rational triangle (neither equilateral nor Pythagorean) with rational Morley triangle gives rise to $r, s, t \in \mathbb{Q}$ satisfying (12). And conversely, $r, s, t \in \mathbb{Q}$ satisfying (12) gives rise to the triangle with edge

$$
\begin{aligned}
a & =2 R \sin A=2 R \sin \frac{A}{3}\left(3-4 \sin ^{2} \frac{A}{3}\right) \\
& =12 R \sqrt{3} \frac{\alpha\left(\alpha^{2}-1\right)\left(\alpha^{2}-9\right)}{\left(\alpha^{2}+3\right)^{3}}
\end{aligned}
$$

(and similarly for $b$ and $c$ ) possessing rational Morley triangles. In terms of $r, s, t$ (up to sign) this triangle is a rational scaling by $12 R \sqrt{3}$ of the triangle with edges

$$
\left\{\frac{r\left(r^{2}-1\right)\left(r^{2}-9\right)}{\left(r^{2}+3\right)^{3}}, \frac{s\left(s^{2}-1\right)\left(s^{2}-9\right)}{\left(s^{2}+3\right)^{3}}, \frac{t\left(t^{2}-1\right)\left(t^{2}-9\right)}{\left(t^{2}+3\right)^{3}}\right\} .
$$

The edges of the Morley triangles are straightforward to compute. For

$$
2 R \sin \frac{A}{3}=\frac{4 \alpha}{\alpha^{2}+3} R \sqrt{3}, \quad 2 R \sin \left(\frac{A+2 \pi}{3}\right)=\frac{-\alpha^{2}-2 \alpha+3}{\alpha^{2}+3} R \sqrt{3},
$$

and

$$
2 R \sin \left(\frac{A-2 \pi}{3}\right)=\frac{\alpha^{2}-2 \alpha-3}{\alpha^{2}+3} R \sqrt{3}
$$

Put 


$$
\begin{aligned}
& \left\{A_{1}, A_{2}, A_{3}\right\}=\left\{\frac{4 \alpha}{\alpha^{2}+3}, \frac{-\alpha^{2}-2 \alpha+3}{\alpha^{2}+3}, \frac{\alpha^{2}-2 \alpha-3}{\alpha^{2}+3}\right\}, \\
& \left\{B_{1}, B_{2}, B_{3}\right\}=\left\{\frac{4 \beta}{\beta^{2}+3}, \frac{-\beta^{2}-2 \beta+3}{\beta^{2}+3}, \frac{\beta^{2}-2 \beta-3}{\beta^{2}+3}\right\}, \\
& \left\{C_{1}, C_{2}, C_{3}\right\}=\left\{\frac{4 \gamma}{\gamma^{2}+3}, \frac{-\gamma^{2}-2 \gamma+3}{\gamma^{2}+3}, \frac{\gamma^{2}-2 \gamma-3}{\gamma^{2}+3}\right\} .
\end{aligned}
$$

Then the edges of the 18 Morley triangles of the triangle at (13) are given by

$$
m_{i j k}=3 R \sqrt{3} A_{i} B_{j} C_{k}, \quad i+j+k \not 1 \bmod 3,
$$

with $\alpha, \beta, \gamma$ as at (11).

The family of triangles (13), subject to (12), is a two-parameter family, and it is interesting to compute the smallest rational triangles represented by the family. Normalizing so that the edges are integers with no common divisor, the examples with edges at most 30000 are the following:

$$
\begin{array}{lll}
\{286,343,343\}, & \{506,2197,2197\}, & \{748,2197,2401\}, \\
\{1254,2197,2401\}, & \{3289,4802,6859\}, & \{4394,4785,6859\}, \\
\{4394,5797,6859\}, & \{4802,6859,7293\}, & \{6630,26411,29791\}, \\
\{6859,6859,10582\}, & \{7889,13718,17493\}, & \{11662,19551,28561\}, \\
\{14858,24167,29791\}, & \{20424,24167,29791\}, & \{26411,28652,29791\} .
\end{array}
$$

For a given triangle $A B C$ with edges $a, b, c$, it is possible to write down the polynomial of degree 18 which gives as roots the edges of the 18 Morley triangles; for this polynomial is simply $\prod(m-8 R \sin A / 3 \sin B / 3 \sin C / 3)$ where the product is over the 18 possibilities for $A / 3, B / 3, C / 3$ which satisfy (2). Computation shows this to be a scalar multiple of the following (note that the coefficients, as was to be expected, are symmetric functions of $\left.a^{2}, b^{2}, c^{2}\right)$ :

$$
\begin{aligned}
& M^{18}-3 M^{16}+12 h M^{15}+3(1-2 g) M^{14}+12 h M^{13} \\
& -\left(1-6 h^{2}+18 g^{2}\right) M^{12}-12 h(1+4 g) M^{11} \\
& -3\left(15 g^{2}-2 g+33 h^{2}\right) M^{10}+4 h\left(1-27 g^{2}-41 h^{2}\right) M^{9} \\
& +3\left(18 g^{3}-3 g^{2}+6 g h^{2}+11 h^{2}\right) M^{8} \\
& +6 h\left(15 g^{2}-2 g+29 h^{2}\right) M^{7} \\
& +\left(81 g^{4}+270 g^{2} h^{2}+321 h^{4}-4 h^{2}\right) M^{6} \\
& +12 h\left(9 g^{3}+11 g h^{2}-2 h^{2}\right) M^{5}+12 h^{2}\left(3 g^{2}-7 h^{2}\right) M^{4} \\
& -48 h^{3}\left(3 g^{2}+5 h^{2}\right) M^{3}-96 g h^{4} M^{2}+64 h^{6}
\end{aligned}
$$




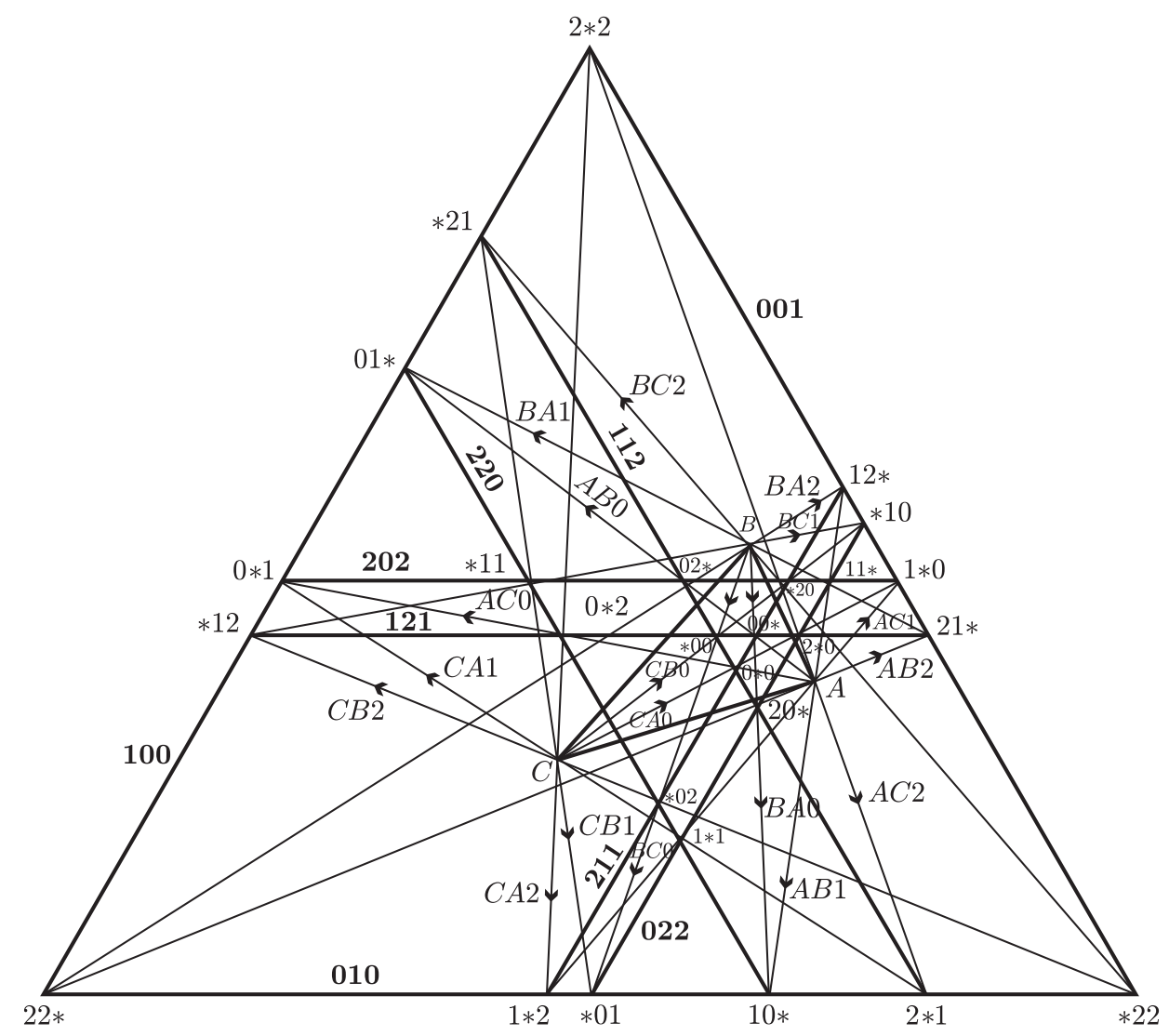

Fig. 4. The triangle $(2401,2197,1254)$ and its 18 Morley triangles

where we set $M=m /(3 R \sqrt{3})$ ( $m$ the Morley edge),

$$
g=\left(a^{2}+b^{2}+c^{2}\right) /\left(162 R^{2}\right) \text { and } h=2 \Delta \sqrt{3} /\left(243 R^{2}\right) .
$$

Corollary. Suppose that $a^{2}, b^{2}, c^{2}$ are not all equal, and do not satisfy $a^{2}+b^{2}=c^{2}$. If the polynomial (14) has one rational root, then all 18 roots are rational.

Finally, it has been observed that if $H$ is the orthocentre (common point of the altitudes) of the triangle $A B C$, then the 72 Morley triangles that arise from the four triangles $A B C, H B C, A H C, A B H$ are homothetic in pairs! It is straightforward to show that, except in the Pythagorean and equilateral cases already noted, if any one of these is rational, then so are all 72 . However, it must be observed that if $A B C$ is rational, with a rational Morley triangle, then the triangles with $H$ as vertex each contain one rational edge, and two edges that are rational multiples of $\sqrt{3}$. Figure 4 illustrates the triangle $(2401,2197,1254)$ with its 18 Morley triangles. The lengths of 
all 72 Morley edges, scaled by a factor 360/91 to make them integers with greatest common divisor 1, are exhibited in the following table:

$\begin{array}{lrrrr} & A B C & H C B & C H A & B A H \\ 000 & 1197 & 305 & 976 & 160 \\ 002 & -6237 & -895 & -2864 & -198 \\ 101 & 5760 & 12980 & 1652 & 836 \\ 102 & -7128 & -19690 & -2506 & -4356 \\ 200 & -2565 & -7015 & -1830 & -3680 \\ 201 & -10800 & -13570 & -3540 & -874 \\ 011 & 8400 & 354 & 10384 & 209 \\ 012 & -10395 & -537 & -15752 & -1089 \\ 110 & 2280 & 4026 & 4697 & 19360 \\ 111 & 9600 & 7788 & 9086 & 4598 \\ 210 & -4275 & -4209 & -10065 & -20240 \\ 212 & 22275 & 12351 & 29535 & 25047 \\ 020 & -3192 & -488 & -6344 & -1040 \\ 021 & -13440 & -944 & -12272 & -247 \\ 120 & -3648 & -10736 & -5551 & -22880 \\ 122 & 19008 & 31504 & 16289 & 28314 \\ 221 & 28800 & 21712 & 23010 & 5681 \\ 222 & -35640 & -32936 & -34905 & -29601\end{array}$

\section{References}

[1] H. S. M. Coxeter and S. L. Greitzer, Geometry Revisited, New Math. Library 19, Singer, 1967.

Department of Mathematics

Arizona State University

Tempe, AZ 85287-1804, U.S.A.

E-mail: bremner@asu.edu

The Computer Laboratory

Cambridge University

Corn Exchange Street

Cambridge CB2 3QG, England

E-mail:mjtg@cus.cam.ac.uk

\author{
12 Bay Terrace \\ Girvan \\ Ayrshire KA26 0AS \\ Scotland \\ Department of Mathematics and Statistics \\ The University of Calgary \\ Calgary \\ Alberta T2N 1N4, Canada \\ E-mail: rkg@cpsc.ucalgary.ca
}

